\title{
Title: Understanding the Social in a Digital Age
}

\author{
Authors: \\ Zoetanya Sujon, University of the Arts London (https://orcid.org/0000-0002-4280-8222) \\ Harry T. Dyer, University of East Anglia (https://orcid.org/0000-0002-1629-730X)
}

\begin{abstract}
Datafication, algorithms, social media and their various assemblages enable massive connective processes, enriching personal interaction and amplifying the scope and scale of public networks. At the same time, surveillance capitalists (Zuboff 2019) and the social quantification sector (Couldry and Mejias 2019) are committed to monetizing every aspect of human communication, all of which threaten ideal social qualities, such as togetherness and connection. This special issue brings together a range of voices and provocations around 'the social', all of which aim to critically interrogate mediated human connection and their contingent socialities. Conventional methods may no longer be adequate, and we must rethink not only the fabric of the social but the very tools we use to make sense of our changing social formations. This special issue raises shared concerns with what the social means today, unpicking and rethinking the seams between digitization and social life that characterize today's digital age.
\end{abstract}

Keywords: social, social media, digital, datafication, algorithms, alternative social media, privacy, assemblages, dark matter, networks

\section{Introduction}

'The social' is increasingly evasive, at once everywhere and nowhere, uniquely human yet entangled with the non-human, internal yet external, straddling and circumventing numerous dichotomies we might seek to better understand it. One area where these tensions have been made overtly visible is the realm of social media. Social media and digital platforms are widely celebrated for connecting more people globally and enabling richer, more dynamic socialities. At the same time, datafication processes are linked to the emptying out of social connection in favour of data accumulation, vanity metrics, algorithmic bias, and surveillance capitalism (Zuboff 2019; Couldry and Mejias 2019). Along with the reshaping of interpersonal relations and intimacies, including the strengthening of historically and systemically disenfranchised communities, new kinds of anti-social collectivities arise in the form of mass trolling, misinformation, rising xenophobia, and political manipulation. We find ourselves in an age shaped not only by 'always-on' connection, but also by the rise of digital empires and increasingly persuasive technologies driven by questionable motives. The social is also not just about interaction. The social is also about the kinds of action made possible and impossible in and through our digital landscapes - and how they are woven together to make up the social fabric. Increasingly the formation of this social fabric is influenced by the enmeshing of users with companies, adverts, marketplaces, medias, and other complex entities, further blurring the boundaries of an easily definable social, emphasising some voices and minimising others. The social in this manner is not just about personal connections and the fuzzy idea of social 'togetherness', as defined by German sociologist, Georg Simmel (1949/1950: 122), but also about the 'the complex interdependencies out of which human life really is made' (Couldry and Van Dijck 205). Traditional public institutions responsible for enculturating and socializing people are increasingly digitized. For example, public information may be less tied to particular sectors and is increasingly decoupled from 
social institutions, impacting how socialization processes relate to the circulation and consumption of information.

We are at a crucial moment, one where it is more important than ever to critically interrogate what it means to be social.

In addition to these big questions about what makes up the social, the digital age raises questions about how to make sense of the social. The continued use of critical traditions in sociology, economics, politics, and media, disciplinary traditions must be balanced with a $21^{\text {st }}$ Century orientation. The digital ecosystem and big data require new techniques for data gathering and analysis, including issue mapping, web-crawling, scraping, trend and metric analysis, among many others (e.g. Rogers 2019). It is also evident that there is a need for a healthy and robust critique of these metrics, logics, and ontologies that big data and algorithms make possible, particularly in how this inform our conceptualisations of the social. In addition, platforms can make big changes, as Facebook did in 2018 by closing down access to its API pages, sparking serious reconsideration of digital research and launching a new era - what Deen Freelon calls 'post API research' (2018). In this sense, the digital provokes and mediates specific socialities, simultaneously reconfiguring how we can research, articulate and understand those specific socialities.

This special issue of New Media and Society responds to these issues, bringing together a range of voices to think through and better understand the social in a digital age. The special issue came together as a result of an interdisciplinary and international conference aimed explicitly at exploring how best to understand, interrogate, and explore the social in a digital age. As organisers we are both active researchers exploring the overlaps between the digital and the social from different disciplines. Zoetanya Sujon's work examines 'the digital' through platforms, social technologies and their complex intersections with everyday life. Harry Dyer's work draws from the field of educational research, broadly exploring how emerging issues of digital culture impact our understanding, practice, and conceptualisation of education. The response to the original conference call was enthusiastic, prompting almost a hundred submissions and highlighting a much wider resonance and interdisciplinary concern with conference theme. The conference, held at the University of East Anglia in January 2019, made clear that there is no single definition or approach to 'the social' - it is multiple, complex, and at once about big processes and small actions.

The articles making up the special issue reflect only a small proportion of work being done on 'the social', and its multiplicities across every dimension of digital life. For example, the social is also about who is represented and how we are both connected and excluded. Sociological approaches to society have long focused on equality and justice, as well as unpacking technological determinisms. While current research identifies the gross and pervasive inequalities bound up in data, algorithms, and long-standing offline hierarchies (e.g. Benjamin 2019a, 2019b; Noble 2018; Eubanks 2018), there is much more to be done in these areas. In addition, sexualities, queerness, identities and intimacies are significant areas of research with a great of the resulting scholarship deeply connected with the social. Although these areas may not be directly reflected in this special issue, they are an essential part of the broader agenda (see for example, Paasonen et al 2019; Skulc 2018; Burgess et al. 2016; Oakley 2016). It is important to continue to challenge the presumption that 'the social' is a singular experience, or that specific manifestations of 'the social' can be normalised as central or neutral narratives, as realities are far more diverse.

Of all the approaches to the social, this special issue surfaces three broad themes. The first of these is around critique, not only of the meaning of the social in relation to datafication (Couldry), algorithmic identity (Kotliar) and privacy within data capitalism (Lutz, Hoffman \& Ranzini), but also of how we are able to think of and articulate the social. The second theme coalesces around work that 
unpacks different manifestations of socialities and digital connections enabled through the affordances, designs, and structures of social media, here exploring the use of 'alternative' platforms (Zulli, Gehl \& Liu), settler counterpublics on Instagram (Karsgaard and MacDonald), the rich digital lives of seemingly isolated youth (Wong), and on the dominance of Western framings in network analysis of the 'right to be forgotten' (Larsen). In the third theme, authors draw from critical feminisms to identify social media's 'sexist assemblages' (Gerrard \& Thornham), the power of humiliation across media to 'oblige' us into social structures (Cefai), and the exclusion of trace publics in 'nodocentric' network analysis (Barnes). These three themes, emerging from the body of work generated beyond and in relation to the conference and special issue, provide an insight into better understanding 'the social' in a digital age. Each of these overlapping themes is addressed more fully below.

\section{Critique and the meaning of the social}

Beginning with an urgent call to reinvigorate the public role of critique, Nick Couldry questions our capacities for understanding datafication and machine learning for our visions of the contemporary and future social world, claiming we have limited conceptual toolkits. Datafication, artificial intelligence and algorithms spark serious anxieties around what it means to be connected, and threaten to replace public knowledge with privatized and monetizable data. The use of risk assessment algorithms like COMPAS (the Correctional Offender Management Profiling for Alternative Sanctions) in US criminal justice systems already points to the 'hollowing out of the decision making capacities of public servants' (Braunis and Goodman 2018 as cited in Couldry, this issue). Offering a big picture view, Couldry provides a schematic overview of key approaches to the social, arguing that despite the legacy inspired by Latour's actor-network theory (ANT) and science and technology studies (STS), these approaches are deeply insufficient. Couldry argues that neither can account for 'embodied agency and reflexive human subjectivity' and their interdependencies with social institutions. Instead, Couldry argues that the 'complexity of social processes' related to datafication demand more sophisticated theoretical resources, as well as a critical accounting of power, agency and their continual recursivities - none of which can occur through ANT's focus on tracing relations. Couldry specifically calls for a focus on the 'larger social order', which can be better conceptualized by drawing from three areas: Norbert Elias' notion of 'figuration' (see also his work with Andreas Hepp 2016); Luc Boltanski's focus on 'definitional power', particularly legal institutions, for making up the world we live in; and a rethinking of Marx's body of work as a social theory of abstraction through capitalism, rather than an economic theory of labour, in line with Moishie Postone's magnum opus (1993). Couldry's 'Recovering Critique in an Age of Datafication' provides a thought-provoking examination of what the social means in a digital age, as well as an impressively broad overview of the conceptual tools interdisciplinary researchers have used to think through the social, concluding with a roadmap for possible future routes for better conceptualising the social.

As if drawing from Couldry's broad conceptualization of 'the social', the next papers focus on specific examples illustrating how the social is deeply intertwined within algorithmic sorting (Kotliar) and the impact of privacy cynicism on German citizens (Lutz, Hoffman \& Ranzini). Employing a 'scavenging ethnography' of Israeli data analytics and mining companies, Dan Kotliar examines the interstices between algorithms and humans in making up identities. Kotliar finds that algorithmic sorting is so granular and hyperindividuated, there is no longer a need for categories - yet, machine generated clusters (e.g. 'cluster 385 ') hold no meaning for data companies, clients, marketers or advertisers. As such, humans rename clusters so algorithmic sorting can be understood by humans, who can then more easily share them across the sector. Kotliar offers an original insight into this process, calling them epistemic amalgams to describe the 'complex blends of algorithmic outputs and human expertise, messy data flows and diverse interpersonal factors'. These factors are all meshed together and 'arbitrarily juxtaposed to quantitative' data through a veneer of logic produced by the 
mathematical nature of algorithms (see also Barnes 2019). As such, identities are computationally deconstructed, and then reconstructed by humans for meaningful human exchange, a process which involves an erasure and 'return of the social'.

Christoph Lutz, Christian Hoffman, and Giulia Ranzini ground their work in privacy studies, identifying the impact of privacy cynicism on the German social fabric within the context of data capitalism. Based on a survey of 1008 people, Lutz, Hoffman and Ranzini contribute quantitative and generalizable findings, arguing that we must rethink the privacy paradox in terms of the rise of privacy cynicism - a term which accounts for the culmination of mistrust, uncertainty, resignation, and especially powerlessness. In doing this, their work explores and reframes assumptions of agency around data sharing practices, adding a much-needed conceptualisation of the growing quantitative data around privacy practices online. This article examines a range of factors involved in the privacy calculus, and furthers understanding of privacy and its complex relationship to 'the social' now and in the future.

Datafication, algorithms, and privacy make up and reshape our experiences of the social, as well as the possibilities to call for public accountability and to generate meaningful social critique. The articles in the next section further examine and unpick the complex interdependencies between the digital and the social.

\section{Connection and socialities in a digital age}

The papers in this section examine particular kinds of platform connections, teasing out the specificities of contingent socialities and their broader implications on 'the social', so often hidden or obscured by power, hegemony, social assumptions, and dominance.

Diana Zulli, Robert William Gehl, and Miao Liu build on alternative media and participatory culture scholarship, using grounded theory to provide an in-depth case study of the alternative social media (ASM) network, Mastodon. In 2017, Mastodon was positioned as a potential contender to overtake Twitter, what the authors refer to as a 'killer hype cycle'. While Mastodon may not yet have done this, Zulli, Gehl and Liu argue that Mastodon enables an alternative kind of sociality, one that is based on a higher level of user engagement, platform control, and meaningful participation, particularly in contrast to corporate social media (CSM). In contrast to abstraction as a central process of datafication, Mastodon does not obfuscate its technical capacities, as CSM's like Facebook and Twitter do, instead Mastodon is built on Free and Open Source Software (FOSS), which is both technically transparent and modifiable by its users. In addition, Mastodon's remarkably smaller scale means it better facilitates intimate connection, within a larger network. Taken together, Mastodon users are able to shape their experience as they would like and this results in a sociality that can be negotiated by its users. Zulli, Gehl and Liu say that this sociality 'challenges the concentrated power of corporate social media'. While Mastodon is not free from problems, like finding appropriate funding and moderating extremism, it does provide insight into what 'the social' might look like if it were divorced from the for-profit motives of corporations and data capitalists which have become normalised and intimately integrated in many social spaces and experiences online.

In 'Picturing the Pipeline', Carrie Karsgaard and Margaret MacDonald present what may be one of the last research projects to scrape data from Instagram's API (closed in December 2018), mapping the contradictory and contested publics on Instagram around Turtle Island's 'proposed Trans Mountain pipeline expansion'. Through scraping thousands of images, hashtags, and keywords, Karsgaard and MacDonald were able to identify the performance of settler colonial discourses made visible in early petro culture posts on Instagram. Based on this, Karsgaard and MacDonald argue that 
'settler colonialism' is not a structure which sits behind Instagram participation, but is instead performed through daily expressions and connective action making up issue publics on Instagram. Notably, popular resistance movements are aligned more with not-in-my-backyard (NIMBY) and colonial pride than indigenous environmental preservation, highlighting multiple public tensions. Karsgaard and MacDonald identify social media as a key site for making and remaking power relations, highlighting the 'discursive potential of Instagram users to both reinforce and unmake' hegemonic and counter publics. In this manner Karsgaard and MacDonald highlight the ongoing contestation of the social that emerges through the enmeshing of users, designs, and discourses.

Rather than focusing on platform specific conceptualizations of the 'social', Mark Wong examines a particular group of users, seemingly isolated young people, challenging what it means to be withdrawn in a digital age where rich online connections may not be visible in face-to-face social behaviours. Commonly known as 'hidden youth' in East Asia, Wong compares the experiences of 32 'hidden' young people in Scotland and Hong Kong (making up 5\% or 41,000 young people in Hong Kong), as a way to develop insight into a much larger phenomenon. Wong finds remarkable similarities across national contexts, including very high levels of social interaction online and via digital media with friends, family and peer groups - and a common preference for this kind of rich and digitally mediated sociality. In fact, youth who prefer online interactions over face-to-face sociality, find they are able to exercise control and are able to choose who, what, and where they interact - providing powerful 'pull' motivations for digital connectivities. Compounding these preferences, Wong also finds that many other factors, such as austerity (Scotland), hypercompetitiveness (Hong Kong), shrinking opportunities, growing labour precarity, general hopelessness towards the future, and social alienation 'push' young people towards an almost entirely online environment. For Wong, it is mistaken to oppose the digital and social as both are mutually constitutive, even if the digital can also provide a safe haven for vulnerable youth.

Rebekah Larsen, resonating with the work of Naomi Barnes (introduced below), calls for a qualitative approach to network analysis, an approach that identifies dominant framings in media coverage of the 'right to be forgotten' (RTBF), as well as identifying who is (in)visible in online networks. Larsen argues that we must re-think network analysis and engage mixed method approaches to better capture the meaning and make-up of the 'networked public sphere', particularly in relation to 'a controversial data protection concept' like 'the right to be forgotten'. For Larsen, network mapping helped reveal 'the most visible framings and sources', showing dominant discourses, dominant legacy sources, and a marginalization of alternative discourses, like the 'right to remember' movement in Latin America. Larsen argues that the fringes of the hyperlink network are important sources for better understanding networks and movements outside of legacy media's well established frames.

These four papers challenge what it is that makes up the social as it is experienced, unfolded, and contested online, not only through the platform specifics of Mastodon and Instagram, but also what it means to be social and where the social can be located in troubled times and with extensive digital networks seamlessly accessible from the bedroom. While these papers explore Mastodon's alternative sociality, colonial and contested hegemony on Instagram, vulnerable youth across digital media, and the (in)visibility of the 'RTBF' across networked media, the next section draws inspiration from critical feminisms to make sense of the social.

\section{Critical feminisms: content, humiliation and dark matter}

The three papers in this final section build upon the need to critically examine how the social manifests itself online, collectively unpacking what assumptions impact the social dynamics and spaces, along with what users, what voices, and what socialities are emphasised and minimised in 
these complex environments. Ysabel Gerrard and Helen Thornham's work begins this discussion through a detailed examination of the practices of content moderation. Gerrard and Thornham propose 'sexist assemblages' as a framework for unpacking the complex mix of gendered assumptions and both human and non-human convergences that inform and enact content moderation online in an ongoing and emerging fashion. Particular attention is paid here to the content generated (or excluded) from searches, content guidelines set by social media platforms, and the algorithmic generation of content, based on data collected from clean browsers and new accounts on Instagram, Pinterest, and Tumblr. This work directly challenges platform claims that social media design is neutral, instead noting that social media platforms frame what it means to be social, minimising and 'othering' some narratives whilst centralising and emphasising others essentially 'representing certain design decisions about how the world is to be ordered' (Bucher 2018 as cited in Gerrard and Thornham, this issue).

In 'Humiliation's Media Cultures: On the Power of the Social to Oblige Us', Sarah Cefai provides a sustained examination of the temporality, location, and affect of humiliation as a 'structure of feeling', one that is increasingly important in the rise of networked media's reputation economy. Drawing from examples like Better Call Saul, Black Mirror and Monica Lewinsky's post-Clinton experience, Cefai examines how humiliation emerges on, in, and through media cultures. Arguing that this nexus is under-explored in queer, feminist, and cultural analysis, Cefai opens up these concepts and their inter-relationships, tracing the history of humiliation to theorize how it works in networked contexts. Cefai argues that humiliation 'casts into action relations between reputation, sense of self, and our capacity to be in the world', going on to suggest that humiliation in digital media cultures places 'the insidious nature of power...hand in hand with the mediated expansion of reputation'. Placing her work within affective studies, Cefai opens up a problematic around the changing nature of humiliation, raising important questions about what it means to be social in a digital age.

Finally, in this section, Naomi Barnes builds a methodological and conceptual framework around the concept of 'trace publics', using this much-needed ontological framing to unpack ways of framing the \#MeToo movement. Her work here critiques network analysis for only accounting for visible nodes in the network - a focus referred to as 'nodocentrism' (Mejias 2010). Barnes aptly calls for attention to be paid to both public and private experiences that leave traces in big data networks. As Barnes notes, there is a large temptation in digital research to pay attention to the 'loud' data that we can draw from sources such as lists, algorithms, posts, hashtags, likes and so on. Her paper makes a clear case for an exploration of the issues a hashtag such as \#MeToo signifies, for a consideration of the absence or removal of data as observed in \#MeToo networks as nodes were deleted when participants removed or changed their posts. Barnes argues we must deeply consider the complexities of a topic, especially one involving sexual violence and multiple vulnerabilities, which may be held together as a cohesive narrative. Like the others in this special issue, Barnes calls for an unpacking of what we count as social, and how we are able to make sense of it. Through a close and sustained consideration of ethics and analysis, Barnes presents a possible path forward for critical and qualitative researchers working in the age of 'big data', and asks for a purposeful and ethical consideration of how we engage with, shape, and present data.

\section{Conclusions: Towards an understanding of the social}

The continued rise and importance of digital media has been framed as somewhat of a crisis, or at the very least, as a necessary consideration for researchers of all backgrounds. The papers included in this special issue raise a number of issues, concerns, and solutions for how we can continue to consider, frame, (re)present, (re)create, and understand the social in a uniquely digital age. This 
special issue presents a number of positions from which we can (un)ravel the digital threads woven into and creating the broad tapestry of 'the social'.

It is evident that there is a need for new approaches to deal with the unique presentations and conceptualizations of data presented in, on, and through digital spaces. As Barnes, Larsen, Kotliar, and other authors in this issue make clear, there is a unique reframing of the social being presented through digital spaces which is often taken as evidence of the social. Further interrogation reveals a dense set of processes and manipulations making up social experience, often reifying human biases, and obscuring what 'the social' is or ought to be. These instances of the social have ongoing and profound impacts, online and offline (see Gerrard and Thornham; and Barnes this issue), and have quickly become key battlegrounds for the construction of discourses that go to the heart of long standing struggles over the framing of social issues (see Karsgaard and MacDonald; Larsen, also in this issue). The ways in which we as researchers respond to these challenges, give voice to them, and choose to analyse them are increasingly important, and we should continue to build and challenge methodological, ontological, and analytical approaches in line with these concerns.

At the same time, the social is made up of both the offline and online, and we must connect the myriad of offline socialities with the framing and experience of the (online) social world. As both Wong's paper and Lutz, Hoffman \& Ranzini's papers make clear here, the ongoing relationships and tensions between, across, and through online and offline spaces present new challenges and opportunities. Larsen and Barnes, in particular, note the complex making of online networks, and call for the critical unmasking of dominant network visibilities, so that we can reveal the power structures shaping our social experience in the digital age. Crucially, it is also apparent that we need not assume that these social experiences are to be left unchallenged. As Zulli, Gehl, and Liu make clear, these (re)presentations of the social we see online can be purposefully undone, and alternate framings can emerge. Cefai's paper, for instance, traces the concept of humiliation as it meets networked media, amplifying the mediation of reputation as a central thread binding us to, through and as part of 'the social'.

It is from this ongoing situation of threaded patterns weaving into other patterns that a path through seems all the more vital, and from which Couldry's call for a re-examination of critique becomes incredibly prescient. There is a temptation to completely rebuild an understanding of the social when faced with digital challenges, such as datafication and algorithmic deconstructions (see Kotliar, this issue). Indeed, there is much to be critical about. Yet, the papers in this special issue also make clear that there are approaches that can be revisited, shedding light on the dense construction of digital socialities and unbundling hopeful possibilities for pathways through. There is no single way to understand the social in a digital age, as the social is not and has never been singular. Instead, the social is an amalgamation of individual and collective action, at once an assemblage of human and non-human, in an ever increasing array of digitally mediated processes and contingent socialities.

\section{Acknowledgements}

We are grateful for the support of our departments and universities, The University of East Anglia for funding and hosting the conference, and to the Media School at London College of Communication, University of the Arts London. In addition, we would like to thank Steve Jones for his guidance and support throughout the process, along with our amazing peer reviewers, whose insights helped refine and develop the papers in this special issue. Lastly, we would like to thank all the authors in the special issue and to those who participated in the conference - as they have helped further our understanding of the social in a digital age. 


\section{Works cited}

Barnes, N (2019) Logic and rhetoric: the problem with digital literacy. Available at: https://medium.com/age-of-awareness/logic-and-rhetoric-the-problem-with-digital-literacy8eaf21db00a7 (accessed 12/11/2019).

Burgess, J; Cassidy, E; Duguay, S; \& Light, B (2016) Making Digital Cultures of Gender and Sexuality with Social Media. Social Media + Society. https://doi.org/10.1177/2056305116672487.

Couldry, N; Mejias, U (2019) The Costs of Connection: How Data is Colonising Human Life and Appropriating it for Capitalism. Stanford: Stanford University Press.

Couldry, N; Hepp, A (2016) The Mediated Construction of Reality. Cambridge, Malden: Polity.

David, G; Cambre, C (2016) Screened Intimacies: Tinder and the Swipe Logic. Social Media + Society. https://doi.org/10.1177/2056305116641976.

Freelon, D (2018) Computational Research in the Post-API Age, Political Communication, 35:4, 665668, DOI: $10.1080 / 10584609.2018 .1477506$.

Mejias, U (2010) The Limits of Networks as Models for Organizing the World. New Media \& Society. 12, pp. 603-617, https://doi.org/10.1177/1461444809341392.

Oakley, A (2016) Disturbing Hegemonic Discourse: Nonbinary Gender and Sexual Orientation Labeling On Tumblr. Social Media + Society, https://doi.org/10.1177/2056305116664217.

Paasonen, S; Light, B; Jarrett, K (2019) The Dick Pic: Harassment, Curation, and Desire. Social Media + Society. https://doi.org/10.1177/2056305119826126.

Plummer, K (2003) Intimate Citizenship: Private Decisions and Public Dialogues. Seattle: University of Washington Press.

Szulc, L (2018) Transnational Homosexuals in Communist Poland: Cross-Border Flows in Gay and Lesbian Magazines. Cham: Palgrave Macmillan.

Zuboff, S (2019) The Age of Surveillance Capitalism: The Fight for a Human Future at the New Frontier of Power. London: Profile Books. 